\title{
DOI 10.31489/2021No4/88-101
}

UDC 530.1

\section{INVARIANT RELATIVISTIC THEORY OF IDEAL GAS}

\author{
Zhumaev M.R. \\ Bukhara Engineering Technological Institute, Uzbekistan, Bukhara, mrjumaev2011@mail.ru
}

\begin{abstract}
The purpose of this study is to develop an original theory of a relativistic ideal gas and to prove the validity of the postulate of the special theory of relativity for the characteristic (i.e., arithmetic mean, root-mean-square) velocities of particles of a relativistic ideal gas even in the massless limit. In this work, the following original methods are used for the first time in the theory of a relativistic ideal gas: the method of nonlinear transformation to prove of the distribution function to find the distribution function of the velocities of particles of a relativistic ideal gas; the equation of state of a relativistic ideal gas was first obtained by averaging the relativistic invariant components of the energy - momentum tensor of a system of noninteracting particles, i.e. ideal gas by the distribution function of the velocities of their particles. The uniqueness and definiteness of the distribution function of the velocities of the particles of a relativistic ideal gas are proved on the basis of the well-known relativistic invariance of the distribution function. For the first time, expressions were obtained for the arithmetic mean and mean square velocities of particles of a relativistic ideal gas. For the first time, a fundamental conclusion is made about the validity of the postulates of the special theory of relativity for the characteristic velocities of particles of a relativistic ideal gas. An equation of state for a relativistic ideal gas is obtained, which relates its pressure, average energy density and temperature.
\end{abstract}

Keywords: distribution function, relativistic ideal gas, arithmetic mean and mean square velocity, equation of state, massless limit.

\section{Introduction}

In the last twenty years, especially on the eve of the celebration of the centenary of the creation of the special theory of relativity by the great A. Einstein, interest in the problems of relativistic statistical physics has sharply increased (see, eg, [1,2] and the literature cited in these works). Naturally, this is due to the need to solve a number of problems in plasma physics [3], relativistic kinetic theory [4,5], super - nonequilibrium relativistic thermodynamics in subatomic physics [6]. It should be noted that such questions arose immediately after the construction of the special theory of relativity. Planck and other classics of physics noted that the Maxwellian distribution of velocities contradicts the fundamental postulate of relativity according to which the speed of particles is limited by the speed of light in emptiness - the limiting speed in nature [7]. The history of generalization of the Maxwellian velocity distribution for the relativistic case lasts more than a hundred years [2,5,7-11]. As the analysis of the literature on this topic shows, the first work in this direction was published by F. Juttner [12]. Despite the fact that the Juttner velocity distribution was the correct relativistic generalization of the Maxwellian velocity distribution (which we will prove below), there is still a serious debate about its correctness and the search for various "modifications" of the Juttner velocity distribution continues. Even in the work of recent years [2,7], continue to obtain, in the language of their authors, curiously surprising results in this regard. Therefore, we have to admit (although this seems unlikely) that the problem formulated at the beginning of the twentieth century remains unsolved. Those no work has proved the validity of the fundamental postulate of the special theory of relativity for the velocities of particles of a relativistic ideal gas. It is clear that for this it is necessary to find the distribution function of the particle velocity of a relativistic ideal gas and, on its basis, to determine the expressions for the characteristic velocities of the particles of a relativistic ideal gas. And then, by passing to the limit, prove that even in the massless limit, these speeds do not exceed the limiting speed in nature.

Another important issue of relativistic statistical physics, as is known, is the derivation of the equation of state for a relativistic ideal gas [7-11] - which establishes a relationship between the pressure, average energy density and temperature of a relativistic ideal gas. In the existing literature, the equation of state for a relativistic ideal gas is obtained thermodynamically [1,4,5,7-12], namely, on the basis of a relativistic generalization of the expression for the free energy or the Gibbs partition function (through which all thermodynamic quantities describing the states of an ideal gas [13, 14]). In our work, we present a new derivation of the equation of state for a relativistic ideal gas by an original method, namely, on the basis of 
averaging the temporal and spatial components of the energy tensor - momentum of a system of noninteracting relativistic (structureless) particles over the velocity distribution function of particles of a relativistic ideal gas. In the context of the problem under consideration, this method seems to us more expedient - a direct method and corresponds to the spirit of Maxwell's own works [15]. Consequently, the proposed theory is a relativistic generalization of the Maxwellian theory of an ideal gas and goes over to the latter in the nonrelativistic limit.

In accordance with the above, the article is organized as follows. In the first section, the distribution function of the particle velocity of a relativistic ideal gas is found using the method of transformation. In the second section, formulas for the mean and root-mean-square velocity of particles of a relativistic ideal gas are obtained. The form of the distribution function of the particle velocity of a relativistic ideal gas in the ultra - relativistic limit is also found here in the case of massless particles (massless particl's). The third section is devoted to a new derivation of the equation of state for a relativistic ideal gas - by averaging macroscopic quantities over the distribution function of the particle velocity of a relativistic ideal gas. In the conclusion, the main conclusions of the proposed theory are presented, as well as their possible applications in high energy physics and relativistic cosmology.

\section{The distribution function of the particle velocity of a relativistic ideal gas}

We will begin the presentation of the proposed theory of a relativistic ideal gas by finding the distribution function of the particle velocity of this gas, since it plays a key role in any statistical system [1315] and, in particular, in the theory of an ideal gas of relativistic particles [1-12]. Figuratively speaking, the distribution function is the cornerstone of kinetic theory.

Note that the desired velocity distribution function can be found in various ways: as a stationary equilibrium solution of the relativistic kinetic Boltzmann equation [2,4,5]; based on the principle of maximum entropy [8-11]. Without discussing here the methods used (they are discussed in more detail in the above literature) and without diminishing the value of these works, we propose the simplest way to determine the velocity distribution function - using the method of transforming the distribution function (see, for example, [18]).

According to this method, on the basis of the known distribution function of the moment of the particles of a relativistic ideal gas $f(\vec{P})=f\left(P_{x}, P_{y}, P_{z}\right)$, we must determine the distribution function of the velocity of particles of a relativistic ideal $\operatorname{gas} \varphi(\vec{v})=\varphi\left(v_{x}, v_{y}, v_{z}\right)$.Here: $P_{x}, P_{y}, P_{z}$ and $v_{x}, v_{y}, v_{z}$ are the components of moments and velocities of particles along the corresponding axes $\mathrm{x}, \mathrm{y}, \mathrm{z}$. Then, based on the method of transformation, we easily obtain the following relation. Using the transition from the moment of particles to their velocities, we obtain:

$$
\int f\left(P_{x}, P_{y}, P_{z}\right) d P_{x} d P_{y} d P_{z}=\int \varphi\left(v_{x}, v_{y}, v_{z}\right) D \mid d v_{x} d v_{y} d v_{z}
$$

Here $\mathrm{D}$ is the transformation determinant defined by the following expression

$$
D=\left|\begin{array}{lll}
\frac{\partial P_{x}}{\partial v_{x}} & \frac{\partial P_{x}}{\partial v_{y}} & \frac{\partial P_{x}}{\partial v_{z}} \\
\frac{\partial P_{y}}{\partial v_{x}} & \frac{\partial P_{y}}{\partial v_{y}} & \frac{\partial P_{y}}{\partial v_{z}} \\
\frac{\partial P_{z}}{\partial v_{x}} & \frac{\partial P_{z}}{\partial v_{y}} & \frac{\partial P_{z}}{\partial v_{z}}
\end{array}\right| .
$$

Note that the determinant of the transformation $D \equiv \frac{\partial\left(P_{x}, P_{y}, P_{z}\right)}{\partial\left(v_{x}, v_{y}, v_{z}\right)}$ will be unique and the same for any type of $\operatorname{DF} f(\vec{P})$ (the form of which we specify below).

Here: $P_{x}, P_{y}, P_{z}$ is defined according to the special theory of relativity by the following expressions:

$$
\rho=P_{x} \stackrel{\rho}{i}+P_{y} \stackrel{\rho}{j}+P_{z} \underset{k}{\rho}=\frac{m \tilde{v}}{\sqrt{1-\frac{v^{2}}{c^{2}}}}, \stackrel{\rho}{\rho}=v_{x} \stackrel{\rho}{i}+v_{y} \stackrel{\rho}{j}+v_{z}{ }_{k} .
$$


Now, taking into account the expression $\mathrm{D}_{1}, \mathrm{D}_{2}$ and $\mathrm{D}_{3}$ (9), we obtain that $D_{1}-D_{2}+D_{3}=\gamma^{2}$. The determinant of the transformation $\mathrm{D}$ from pulses $P_{x}, P_{y}, P_{z}$ to velocities $v_{x}, v_{y}, v_{z}$ is finally determined by the following expression $[2,24,25]$ :

$$
D=m^{3} \gamma^{5}=m^{3}\left(1-\frac{v^{2}}{c^{2}}\right)^{-\frac{5}{2}}
$$

Note that the found determinant of the transformation $\mathrm{D}$ from momenta to velocity remains valid for an arbitrary distribution function of momenta of particles of a relativistic ideal gas. In particular, for quantum relativistic ideal gases, the particles of which obey, as is well known to the statistics of Fermi-Dirac and Bose-Einstein [19].

Before proceeding to the definition of the distribution function of the velocities of the particles of a relativistic ideal gas, I would like to bring the following transformation of the elementary volume from the space of momenta to the space of velocities (which follows from (1):

$$
d P_{x} d P_{y} d P_{z}=D d v_{x} d v_{y} d v_{z}
$$

Hence, in particular, it follows that

$$
P^{2} d P=D v^{2} d v
$$

which is easy to verify, given that

$$
P=\frac{m v}{\sqrt{1-\frac{v^{2}}{c^{2}}}}, \quad \frac{d P}{d v}=\frac{m}{\left(1-\frac{v^{2}}{c^{2}}\right)^{\frac{3}{2}}} .
$$

Now let us determine the distribution function of the velocity of particles of a relativistic ideal gas using the Boltzmann distribution for the momenta of particles of a relativistic ideal gas [18]:

$$
f(\rho)=f\left(P_{x}, P_{y}, P_{z}\right)=B \exp \left[-\frac{\sqrt{E_{0}^{2}+p^{2} c^{2}}}{k T}\right] .
$$

where $E_{0}=m c^{2}$ is the rest energy of a gas particle, $\mathrm{kT}$ is thermal energy, B is a constant, which is determined, as always, by the normalization condition for the distribution function, will be found below.

Further, taking into account that the momentum of a relativistic particle $\vec{P}$ is related to its velocity $\vec{v}$ in a nonlinear manner, according to expression (3)

$$
E=\sqrt{E_{0}^{2}+p^{2} c^{2}}=m c^{2}\left(1-\frac{v^{2}}{c^{2}}\right)^{-\frac{1}{2}},
$$

based on the DF of the moments $(8) f(\vec{P})(14)$, we find the distribution function of the velocity vector of the particles of the relativistic ideal gas

$$
\varphi\left(v_{x}, v_{y}, v_{z}\right)=B \exp \left[-b\left(1-\frac{v^{2}}{c^{2}}\right)^{-\frac{1}{2}}\right], \quad b=\frac{m c^{2}}{k T}
$$

Therefore, the probability that the speed lies in the interval $(\hat{v}, \hat{v}+d \hat{v})$ or that, the same - the components of the velocity in the interval $\left(v_{x}, v_{x}+d v_{x}\right),\left(v_{y}, v_{y}+d v_{y}\right)$ and $\left(v_{z}, v_{z}+d v_{z}\right)$ is determined according to (10) as follows

$$
d W=\varphi\left(v_{x}, v_{y}, v_{z}\right) d v_{x} d v_{y} d v_{z} .
$$

Finally, in the conclusion of the section, we find the distribution function of the modulus of particle velocities based on the formula for transforming the distribution function from $f(\not)$ to $f(k)$ (1) and formula (6), taking into account expressions (3) and (10): 


$$
\begin{aligned}
& \int f(\rho) d \rho=\int \varphi(\rho) d \rho=B \cdot 4 \pi \int_{0}^{c} m^{3}\left(1-\frac{v^{2}}{c^{2}}\right)^{-\frac{5}{2}} . \\
& \cdot v^{2} \exp \left[-b\left(1-\frac{v^{2}}{c^{2}}\right)^{-\frac{1}{2}}\right] d v \equiv \int_{0}^{c} F(v) d v .
\end{aligned}
$$

Thus, the distribution function of the velocity modulus of particles of a relativistic ideal gas is determined by the following expression

$$
F(v)=4 \pi m^{3} v^{2}\left(1-\frac{v^{2}}{c^{2}}\right)^{-\frac{5}{2}} \exp \left[-b\left(1-\frac{v^{2}}{c^{2}}\right)^{-\frac{1}{2}}\right] \cdot B .
$$

Further, introducing the normalized particle velocity $u=\frac{v}{c}$, we can find on the basis of (13) the reduced distribution function of the modulus of the normalized particle velocity $F(u)$

$$
\int_{0}^{c} F(v) d v=B \cdot 4 \pi(m c)^{3} \int_{0}^{1} F(u) d u
$$

Here

$$
F(u)=u^{2}\left(1-u^{2}\right)^{-\frac{5}{2}} \exp \left[-b\left(1-u^{2}\right)^{-\frac{1}{2}}\right] .
$$

Now we find the constant B using the normalization condition for the distribution function of the velocity moduli of particles of a relativistic ideal gas $F(v)$

$$
\int_{0}^{c} F(v) d v=B \cdot 4 \pi(m c)^{3} \int_{0}^{1} F(u) d u=1 .
$$

Therefore, the constant B according to (14) - (16) is determined by the expression

$$
B=\frac{1}{4 \pi(m c)^{3}} \cdot \frac{b}{k_{2}(b)} \text {. }
$$

Thus, the reduced distribution function of the moduli of the normalized velocities of particles of a relativistic ideal gas can be written in the following compact form:

$$
\Phi(u)=\frac{b F(u)}{k_{2}(b)} \equiv \frac{b}{k_{2}(b)} u^{2}\left(1-u^{2}\right)^{-\frac{5}{2}} \exp \left[-b\left(1-u^{2}\right)^{-\frac{1}{2}}\right] .
$$

The distribution function of the velocity modulus of particles of an ideal gas found by us can be used to calculate the cross section for collisions of particles in a relativistic ideal gas [20] and the cross section for reactions of ultrarelativistic particles in subatomic physics [6]. It may be of interest in studies of the relativistic ionization of atoms by high-energy gas particles, as well as in the field of relativistic laser spectroscopy.

At the end of the section, we present a proof of the invariance of the particle velocity distribution functions of a relativistic ideal gas. The proof given here of the derivation of the velocity distribution function of particles of a relativistic ideal gas MB is final. This follows from the fact that the relativistic Boltzman distribution function for moments is relativistic invariant. Indeed, taking into account that the fourdimensional momentum of gas particles is

$$
P^{i}=m v^{i}=m\left(\frac{c}{\sqrt{1-\frac{v^{2}}{c^{2}}}}, \frac{v}{\sqrt{1-\frac{v^{2}}{c^{2}}}}\right)
$$

and the four-dimensional velocity of the rest coordinate system $(\mathrm{V}=0)$ is

$$
V^{i}=(c, 0)
$$

we get that 


$$
P^{i} v=\frac{m c^{2}}{\sqrt{1-\frac{v^{2}}{c^{2}}}}=\sqrt{\left(m c^{2}\right)+P^{2} c^{2}}
$$

Since the scalar product of any four-dimensional vectors is invariant, any equilibrium distribution possesses this property.

Obviously, the total probability

$$
\int f\left(P_{1}, P_{2}, P_{3}\right) d P_{1} d P_{2} d P_{3}=1
$$

does not depend on the transformation of the distribution function, which was used to find the relativistic velocity distribution function.

Let us assume that the momentum distribution function of particles of a relativistic ideal gas (8) is invariant if the relation is invariant

$$
\frac{i_{0}}{T_{0}}=\frac{i}{T}
$$

Here $i_{0}=i=p_{\mu} v^{\mu}$ - according to the above, it is an invariant-as a scalar product of the vectors $\mathrm{p}_{\mu}$ and $\mathrm{v}^{\mu}$ and, as is known, does not change under Lorentz transformations.

Hence, we get that the condition $\mathrm{T}_{0}=\mathrm{T}$ must be fulfilled, i.e. the temperature of the RIC is the same in all Inertial System Frame (ISF). Thus, the main drawback of the existing non-invariant RIG theories is eliminated, according to which, during Lorentz transformations, both the DF and the macroscopic characteristics of the RIG change - which contradicts the invariance of the laws of nature in all ISF.

Summarizing the above, we will come to the fundamental conclusion that during the transition from one ISF to another, the statistical properties of the RIG do not change: neither the velocity distribution function nor the equation of state of the RIG do not change under Lorentz transformations, i.e. they are invariant in all ISF. Naturally, this also holds true for quantum rigs.

\section{Characteristic velocities of particles of a relativistic ideal gas}

Based on the distribution function of the velocity module of particles of a relativistic ideal gas found in the previous section, it is possible to find any macroscopic characteristic of this gas depending on the velocity based on the formula for calculating the mean

$$
<G>=\int_{0}^{C} G(v) F(v) d v
$$

The quantity included in this expression, as is known, gives the probability of finding the particle velocity modulus in the velocity interval $[v, v+d v]$

$$
d W=F(v) d v .
$$

Then, using the explicit form of the relativistic velocity distribution $F(v)(19)$ and the definition of the function $\Phi(u)$ (17), we obtain the following expression to find the above mentioned probability

$$
d W=B \cdot 4 \pi m^{3} v^{2}\left(1-\frac{v^{2}}{c^{2}}\right)^{-\frac{5}{2}} \exp \left[-b\left(1-\frac{v^{2}}{c^{2}}\right)^{-\frac{1}{2}}\right] d v=\Phi(u) d u
$$

Here $\Phi(u)$ is the reduced distribution function of the modulus of the normalized particle velocities determined according to (17), by the formula

$$
\Phi(u)=\frac{d W}{d U}=\frac{b}{k_{2}} u^{2}\left(1-u^{2}\right)^{-\frac{5}{2}} \exp \left[-b\left(1-u^{2}\right)^{-\frac{1}{2}}\right] .
$$

However, according to the Maxwell distribution, which is obtained from the relativistic distribution (13) in the limit $k T<<m c^{2}$ and $v<<c$, i.e. in the nonrelativistic approximation, the probability that the modulus of the particle's velocity belongs in the velocity range $[v, v+d v]$ is determined by the expression [15]

$$
d W=F_{M}(v) d v=\frac{4}{\sqrt{\pi}} \cdot u^{2} \exp \left(-u^{2}\right) d u=\Phi_{M}(u) d u
$$


Here $u$ is the particle velocity normalized to the most probable velocity $v_{m p}=\sqrt{\frac{2 k T}{m}}$ i.e. $u=\frac{v}{v_{m p}}$. Comparing the relativistic distribution function $\Phi(u)$ with the Maxwellian distribution function $\Phi_{M}(u)$, we come to the conclusion that, unlike the latter, the first distribution cannot be represented in a single universal form for all gases. The reason for this difference, as can be seen from the comparison of the two distributions, is the presence of the parameter $b$ in the relativistic distribution, which is equal to the ratio of the rest energy of gas particles to the thermal energy kT. In particular, as we will prove below, it is this circumstance that leads to the fact that for a relativistic ideal gas the law of uniform distribution of the average kinetic energy over the degrees of freedom does not hold. Attention was drawn to this, for example, in Pauli's book [21].

The unusualness of the relativistic distribution function (or what, the same thing, the relativistic distribution) $\Phi(u)$ makes us be more attentive to this attractive person (it is clear that such people are extremely rare, if not completely!). Of course, we are primarily interested in, as in the case of the Maxwellian distribution, the characteristic velocities of the relativistic distribution, namely: the most probable, mean and root-mean-square velocities of particles of a relativistic ideal gas.

The most probable particle velocity corresponds to the maximum of the relativistic velocity distribution function $F(v)$ or the relativistic distribution function $\Phi(u)$. It is determined by the extremum condition $\frac{d F(v)}{d v}=0$, which is equivalent to condition $\frac{d \Phi(u)}{d u}=0$. Calculating this derivative and making a number of simplifications, we obtain the following equation

$$
\left[2+\frac{u^{2}}{1-u^{2}}\left(5-\frac{b}{\sqrt{1-u^{2}}}\right)\right] \Phi(u)=0 .
$$

Further, taking into account that for the values of the parameter $b$ other than zero: $\Phi(u)=0$ at $u=0$ and $u=1$, we come to the conclusion that these values of the velocities correspond to the zeros of the relativistic distribution.

In addition to these roots, equation (23) also has intermediate roots, as follows from it, determined by the solution of the following equation

$$
b u^{2}=\left(2+3 u^{2}\right) \sqrt{1-u^{2}}
$$

We further restrict ourselves to analyzing its solutions only for very large and very small values of the parameter $b^{1}$.

As follows from equation (24), for any finite values of $b$, its roots belong to the interval of normalized velocities $0<u<1$.

For $u<<1$, which corresponds to nonrelativistic particles, from (24) we obtain that if $b>>1$ or $\kappa T<<m c^{2}$, it has the following approximate solution

$$
u_{m p}=\frac{v_{m p}}{c}=\sqrt{\frac{2}{b}}=\sqrt{\frac{2 k T}{m c^{2}}}
$$

Therefore, as expected, in this nonrelativistic limit we obtain a result following from the Maxwellian distribution of $F_{M}(v)$ or $\Phi_{M}(u)$ - which is valid in this case.

Now we find a solution to equation (24) close to the limiting one, i.e. $u=1-\varepsilon(0<\varepsilon<<1)$. Then it follows from this equation that

$$
u \approx 1-\frac{b^{2}}{50} .
$$

This solution corresponds to very small values of the parameter $b<<1$, which means an ultrarelativistic limit. Further, taking into account the asymptotic behavior of the modified second-order Bessel function $\kappa_{2}(b) \approx \frac{2}{b^{2}}$, according to (21), we come to the conclusion that the maximum of DF $\Phi(u)$ is described by the following expression

\footnotetext{
${ }^{1}$ We will not give general solutions to this equation because of its cumbersomeness.
} 


$$
\Phi_{\text {макс }} \approx \frac{2}{b^{2}} \text {. }
$$

Therefore, in contrast to the Maxwell distribution $\Phi_{M}(u)(22)$, which has a maximum at $u_{m p}=1$ at any value of $b$, the relativistic distribution $\Phi(u)(21)$ has a maximum value depending on the parameter $b$. In particular, at $b<<1$, according to (27), the maximum of this distribution grows, i.e. the relative number of particles grows with velocities close to the limiting $u \approx 1$. However, as is known, the maximum of the Maxwellian distribution $F_{M}(v)$ decreases with increasing temperature, i.e. tends to zero at very high temperatures. This is the main difference between the relativistic distribution $F(v)$ and the Maxwelliandistribution $F_{M}(v)$ !

We now turn to determining the average velocity of particles of a relativistic ideal gas. According to the formula for calculating the means (18), we obtain that

$$
<v>=\int_{0}^{C} v F(v) d v=c \int_{0}^{1} u \Phi(u) d u=c<u>
$$

Using expression (28), we obtain the following formula for the average velocity of particles of a relativistic ideal gas $[2,24,25]$ :

$$
<u>=\frac{2 e^{-b}}{k_{2}(b)}\left(\frac{1+b}{b^{2}}\right) .
$$

In a similar way, we determine the mean square of the velocity again using the formula for calculating the means (18), according to which

$$
<v^{2}>=\int_{0}^{C} v^{2} F(v) d v=c^{2} \int_{0}^{1} u^{2} \Phi(u) d u=c^{2}<u^{2}>.
$$

Using (21) and (30), we obtain the following formula for the mean square of the normalized velocity of particles of a relativistic ideal gas [2, 24, 25]

$$
<u^{2}>=1-\frac{k_{1}(b)-k i_{1}(b)}{k_{2}(b)} b .
$$

The found expressions for the mean modulus and the mean square of the modulus of the normalized particle velocity (38), (39) allow us to determine another most important characteristic of the relativistic distribution (27) - the root-mean-square fluctuation of the velocity of gas particles:

$$
\left.D v=\left\langle v^{2}>-<v\right\rangle^{2}=c^{2}\left[<u^{2}>-<u\right\rangle^{2}\right]=c^{2} D u,
$$

which describes the characteristic spread of the velocities of particles of a relativistic ideal gas.

Now let us analyze the behavior of the found characteristic velocities for a very large value of the parameter b, i.e. $b>>1$. In this case, as already noted, the thermal energy $k T$ is much less than the rest energy of the particles $m c^{2}$. Further, taking into account the asymptotics of $k_{1}(b)$ and $k i_{1}(b)$ [22]:

$$
\begin{aligned}
& k_{1}(b) \approx \sqrt{\frac{\pi}{2 b}} e^{-b}\left(1+\frac{3}{8 b}-\frac{15}{2} \cdot \frac{1}{(8 b)^{2}}+\ldots\right), \\
& k i_{1}(b) \approx \sqrt{\frac{\pi}{2 b}} e^{-b}\left(1-\frac{5}{8 b}+\frac{129}{128} \cdot \frac{1}{b^{2}}+\ldots\right),
\end{aligned}
$$

in the considered limit, we obtain

$$
\left[k_{1}(b)-k i_{1}(b)\right] b \approx \sqrt{\frac{\pi}{2 b}} e^{-b}\left(1-\frac{9}{8 b}\right) .
$$

Now recalling the asymptotics of the modified second-order Bessel function $k_{2}(b)$

$$
k_{2}(b) \approx \sqrt{\frac{\pi}{2 b}} e^{-b}\left(1+\frac{15}{8 b}+\ldots\right),
$$

on the basis of expressions (30), (35), (36) we obtain the following formula for the mean square of the velocity of ideal gas particles 


$$
<v^{2}>\approx c^{2} \cdot \frac{3}{b}\left(1-\frac{45}{(8 b)^{2}}\right) \approx \frac{3 \kappa T}{m} .
$$

This is a result following from the Maxwellian distribution for the mean square of the particle velocity in the nonrelativistic limit.

In the same limit, in accordance with expressions (28), (29), and (36), we obtain

$$
<v>\approx c \cdot 2 \sqrt{\frac{2}{\pi}} \cdot \frac{1}{\sqrt{b}} \approx \sqrt{\frac{8}{\pi} \cdot \frac{\kappa T}{m}} .
$$

This is the formula for the average velocity of the Maxwellian distribution. Thus, within the framework of the applicability of the Maxwellian distribution, the root-mean-square fluctuation of the velocities of particles of an ideal gas according to (32), (37), and (38) is determined by the following expression

$$
D v=c^{2} D u \approx c^{2}\left(3-\frac{8}{\pi}\right) \frac{1}{b} \approx\left(3-\frac{8}{\pi}\right) \frac{k T}{m} .
$$

Finally, consider the case of ultrarelativistic (or massless) particles, which corresponds to very small values of the parameter b, i.e. $b<<1$ (or $b \rightarrow 0$ ). Then, taking into account the asymptotics of the functions $k_{1}(b), k_{2}(b)$ and $k i_{1}(b)$ in this limit [22]

$$
k_{1}(b) \approx \frac{1}{b}, \quad k_{2}(b) \approx \frac{2}{b^{2}}, \quad k i_{1}(b) \approx \frac{\pi}{2},
$$

we obtain from (29) and (31) the following results

$$
<u>=1-b^{2}, \quad<u^{2}>\approx 1-\frac{1}{2} b^{2} .
$$

The latest results mean that as $\mathrm{m} \rightarrow 0$, these characteristic speeds tend to the speed of light $\mathrm{c}$.

Consequently, in the ultrarelativistic limit, the root-mean-square fluctuation of the velocities of particles of an ideal gas is determined by the following formula

$$
D v=c^{2} D u \approx c^{2} \frac{3}{2} b^{2} \approx \frac{3}{2} c^{2}\left(\frac{m c^{2}}{\kappa T}\right)^{2} .
$$

Thus, in this section, on the basis of the relativistic distribution of velocities (19), it is proved that the characteristic velocities of gas particles: the most probable, mean and root-mean-square velocities do not exceed the speed of light. In particular, taking into account the unattainability of absolute zero temperature, proved by Nernst, we come to the conclusion that only massless particles at any temperature have speeds equal to the speed of light. In addition, it is possible to show the boundedness of the root-mean-square fluctuations of particle velocities for any value of the parameter $b$. Concluding the section, we note that these qualitative considerations in obtaining solution (24) can be easily obtained by finding the values of $b$ corresponding to the most probable velocities $u_{m p}=\frac{v_{m p}}{c}$ which are determined as follows:

$$
b=\left(3+\frac{2}{u^{2}}\right) \sqrt{1-u^{2}} .
$$

From here, for $u<<1$ and $u \approx 1-\varepsilon \quad(0<\varepsilon<<1)$, we obtain approximate solutions (25) and (26) of equation (24).

Based on the above analyzes, we come to the conclusion that in the massless limit all characteristic particle velocities tend to the speed of light. In other words, the distribution function of the velocity modulus of particles of an ultra relativistic ideal gas becomes delta-shaped $F_{0}(\vartheta)=\delta(\vartheta-c)$, and their directions are completely random and equally probable, i.e. distributed isotropically. Indeed, the relativistic distribution function of the normalized velocity $\Phi(u)$ at $b \ll 1$ takes the following form

$$
\Phi(u) \approx \frac{b^{3}}{2} \cdot u^{2}\left(1-u^{2}\right)^{-\frac{5}{2}} \exp \left[-b\left(1-u^{2}\right)^{-\frac{1}{2}}\right] .
$$

Further, setting $u^{2}=1-\varepsilon^{2}(\varepsilon \rightarrow 0)$, weobtain 


$$
\Phi(u) \approx \frac{b^{3}}{2 \varepsilon^{5}} \exp \left(-\frac{b}{\varepsilon}\right) .
$$

Hence it is clear that if $b=\sqrt{\varepsilon}$, then at $\varepsilon \rightarrow 0$ the function $\Phi(u)$ tends exponentially to zero. If $b=\varepsilon^{\frac{3}{2}}$, then at $b \rightarrow 0 \Phi(u)$ becomes infinite. Therefore, in this case, the reduced distribution function will be delta-shaped, i.e.

$$
\Phi(u)=\delta(1-u)(\text { fig. } 1)
$$

Thus, based on the analysis of the expressions for the characteristic velocities of the particles of a relativistic ideal gas, we come to the fundamental conclusion that the most probable average androot-meansquare velocity does not exceed the speed of light - the limiting velocity in nature. This proves the validity of the postulates of the special theory of relativity in relativistic statistical physics.
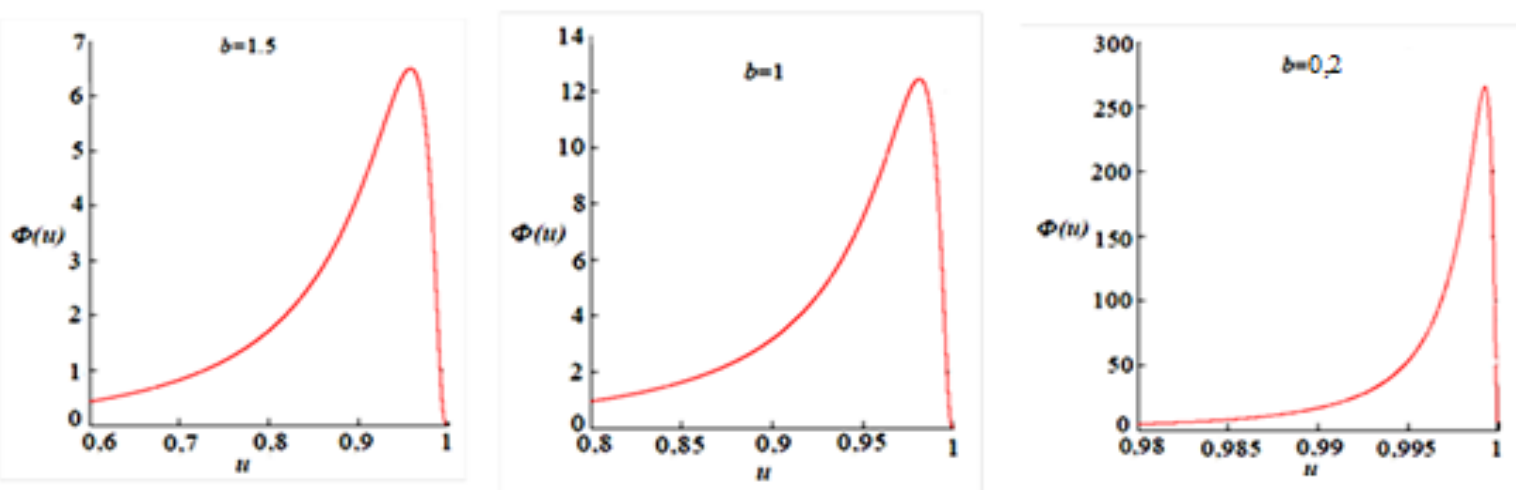

Fig.1. The distribution function of the moduli of the normalized velocities of the particles of a relativistic ideal gas $(\mathrm{b}=1.5 ; 1 ; 0.2)$

As an example, we find the root-mean-square velocity of the particles of a relativistic ideal gas at the value of the parameter, when the thermal energy is equal to one third of the rest energy of the gas particle.

Using formula (31) and taking into account the values of the special functions appearing in it, we obtain

$$
\vartheta_{\kappa \beta} \equiv \sqrt{\left\langle\vartheta^{2}\right\rangle} \approx 0.515 c \text {. }
$$

However, according to Maxwell's formula for the mean square velocity (37) $\vartheta_{\kappa b}=c$. This result has no physical meaning, since, according to the special theory of relativity, no particle with a nonzero mass can have a speed equal to the speed of light. The latter conclusion also follows from the relativistic distribution function of the particle velocity modulus found by us: if $m \neq 0$, then at $\vartheta \rightarrow c$ the function $F(\vartheta) \rightarrow 0$.

\section{Equation of state for a relativistic ideal gas}

As is known, the properties of any ideal gas are determined by its equation of state, which connects three thermodynamic quantities: pressure, average energy density and temperature [13,23]. Therefore, our goal in this section is to determine the equation of state for a relativistic ideal gas. Before proceeding with the direct solution of this problem, we give a short proof of expressions for the average energy density and pressure of a relativistic ideal gas. To do this, we use the following well-known expression for the energymomentum tensor of a system of noninteracting particles [23]:

$$
T^{i k}=n m c<\frac{d x^{i}}{d t} \cdot \frac{d x^{k}}{d S}>, d S=c \sqrt{1-\frac{v^{2}}{c^{2}}} d t
$$

where $n$ is the number of particles per unit volume, and the angle brackets denote averaging over the velocity distribution function of gas particles.

Further, taking into account the definitions of the four-dimensional radius vector of the particle $x^{i}$

$$
x^{i}=\left(x^{0}, x^{1}, x^{2}, x^{3}\right)=(c t, x, y, z)=\left(c t, r^{\rightarrow}\right),
$$

we find the following expressions necessary to find $T^{i k}$ : 


$$
\begin{aligned}
& \frac{d x^{i}}{d t}=\left(c, v^{\rightarrow}\right)=\left(c, v_{x}, v_{y}, v_{z}\right), \\
& \frac{d x^{k}}{d S}=\left(\frac{1}{\sqrt{1-\frac{v^{2}}{c^{2}}}}, \frac{v^{\rightarrow}}{c \sqrt{1-\frac{v^{2}}{c^{2}}}}\right),
\end{aligned}
$$

where $v^{\rightarrow}$ is the usual three-dimensional particle velocity. Therefore, using expressions (43) - (46), one can determine any component of the energy-momentum tensor $T^{i k}$ of a system of noninteracting particles, for example,

where $\alpha=x, y, z$.

$$
T^{00}=n m c^{2}<\frac{1}{\sqrt{1-\frac{v^{2}}{c^{2}}}}>, \quad T^{\alpha \alpha}=n m<\frac{v_{\alpha}^{2}}{\sqrt{1-\frac{v^{2}}{c^{2}}}}>,
$$

Hence it follows that the time component of the energy-momentum tensor $T^{00}$ is equal to the average energy density $\rho$ of the relativistic ideal gas, determined by the expression

$$
\rho=n m c^{2}<\frac{1}{\sqrt{1-\frac{v^{2}}{c^{2}}}}>
$$

Now, using the spatial components of the energy-momentum tensor, we obtain that

$$
T^{x x}+T^{y y}+T^{z z}=n m c^{2}<\frac{v^{2}}{c^{2} \sqrt{1-\frac{v^{2}}{c^{2}}}}>.
$$

However, for any isotropic distribution of particle velocities (otherwise it cannot be due to the equality of all directions in space)

$$
T^{x x}=T^{y y}=T^{z z}=P,
$$

Thus, from $\left(47^{*}\right)$ and (49) we obtain the following expression for the pressure of a relativistic ideal gas:

$$
P=\frac{n m c^{2}}{3}<\frac{v^{2}}{c^{2} \sqrt{1-\frac{v^{2}}{c^{2}}}}>
$$

We emphasize that we did not cite here the expressions for the mixed components of the energymomentum tensor $T^{i k}$ when $i$ and $k$ are not equal, simply because they are all equal to zero. Now using the identity

$$
\frac{1}{\sqrt{1-u^{2}}}=\sqrt{1-u^{2}}+\frac{u^{2}}{\sqrt{1-u^{2}}}, \quad u=\frac{v}{c},
$$

and taking into account expressions (48) and (50), we obtain the following important equality, which establishes a relationship between the pressure $P$ and the average energy density $\rho$ of a relativistic ideal gas

$$
\rho-3 P=n m c^{2}<\sqrt{1-\frac{v^{2}}{c^{2}}}>
$$

Thus, as follows from the expressions for the average energy density $\rho$ (48) and pressure $P(50)$, as well as the equation of state (52) connecting them, they are all determined by statistical averaging based on the relativistic distribution of the velocities of their particles $F(v)$ (13). This is the originality of the method used by us for the first time for finding the indicated macroscopic quantities of a relativistic ideal gas.

Let's start solving this problem by determining the average energy density of a relativistic ideal gas based on expressions (48) and (13): 


$$
\rho=n m c^{2} \int_{0}^{C} \frac{1}{\sqrt{1-\frac{v^{2}}{c^{2}}}} F(v) d v=n m c^{2} \int_{0}^{1} \frac{1}{\sqrt{1-u^{2}}} \Phi(u) d u
$$

Now, applying the substitution $u=t h x(0<x<\infty)$ and taking into account expression $\Phi(u)$ (21), we obtain

$$
\rho=n m c^{2} \frac{b}{k_{2}(b)}\left(\int_{0}^{\infty} s h^{2} x e^{-b c h x} d x+\int_{0}^{\infty} s h^{4} x e^{-b c h x} d x\right)
$$

The integrals included in (54) are respectively equal [22]

$$
I_{1}=\frac{k_{1}(b)}{b}, \quad I_{2}=3 \frac{k_{2}(b)}{b^{2}},
$$

where $k_{1}(b)$ and $k_{2}(b)$ are the modified Bessel functions of the first and second order.

Thus, for the average energy density $\rho$ of a relativistic ideal gas, we obtain the following beautiful expression

$$
\rho=n m c^{2}\left[\frac{k_{1}(b)}{k_{2}(b)}+\frac{3}{b}\right], \quad b=\frac{m c^{2}}{\kappa T} .
$$

Next, we calculate the right-hand side of equality (52)

$$
n m c^{2}<\sqrt{1-\frac{v^{2}}{c^{2}}}>=n m c^{2} \frac{b}{k_{2}(b)} \int_{0}^{\infty} s h^{2} x e^{-b c h x} d x .
$$

Now, taking into account the first integral in expression (55), from this we obtain

$$
n m c^{2}<\sqrt{1-\frac{v^{2}}{c^{2}}}>=n m c^{2} \frac{k_{1}(b)}{k_{2}(b)} .
$$

Substituting the found mean in the right-hand side of equality (52), we find the following expression for the pressure of the Maxwell-Boltzmann relativistic ideal gas

$$
P=n k T \text {. }
$$

Of course, this result can also be obtained by direct averaging of the expression for pressure (50). However, now, unlike the nonrelativistic ideal gas theory, the pressure of a relativistic ideal gas will not be directly proportional to the average kinetic energy of gas particles $\left\langle E_{k}\right\rangle$, since, according to (56)

$$
\left\langle E_{k}\right\rangle=m c^{2}\left[\frac{k_{1}(b)}{k_{2}(b)}+\frac{3}{b}-1\right],
$$

is a nonlinear function of the thermal energy $\mathrm{kT}$ and the rest energy of the gas particle $\mathrm{mc}^{2}$.Inparticular

$$
\frac{\left\langle E_{k}\right\rangle}{k T}=b \cdot\left[\frac{k_{1}(b)}{k_{2}(b)}+\frac{3}{b}-1\right]
$$

Using this formula, by measuring $\left\langle E_{k}\right\rangle$ and $T$, you can determine the rest energy of gas particles $\mathrm{mc}^{2}$ in a non-trivial way. Thus, the equation of state of a relativistic ideal gas connecting its pressure, average energy density and temperature is determined by the following expression

$$
\rho-3 P=n m c^{2} \frac{k_{1}(b)}{k_{2}(b)}, \quad b=\frac{m c^{2}}{\kappa T} .
$$

We emphasize that the above results can be obtained by other statistical methods, which were mentioned at the beginning of the first section.

Let us now investigate the asymptotic behavior of the pressure and average energy density of a relativistic ideal gas at the limiting values of the parameter $\mathrm{b}$. If the thermal energy $\mathrm{kT}$ is negligible compared to the rest energy of the gas particles $m c^{2}$, then the parameter $b>>1$. Further, taking into account the asymptotics of the functions $k_{1}(b)$ and $k_{2}(b)$ (36), we have 


$$
\frac{k_{1}(b)}{k_{2}(b)} \approx 1-\frac{3}{2 b}-\frac{45}{64 b^{2}} .
$$
formula

Then it follows from (56) that in this limit the mean energy density is determined by the following

$$
\rho \approx n m c^{2}+\frac{3}{2} n k T
$$

However, M. Consequently, only for a nonrelativistic ideal gas, the law on the uniform distribution of the average kinetic energy over the degrees of freedom is valid, i.e.

$$
<E_{k}>=\frac{3}{2} \kappa T \text {. }
$$

In the opposite limit $b<<1$ (which means not only $T \rightarrow \infty$, but also $m \rightarrow 0$ !), The modified Bessel functions of the first and second order have the asymptotics indicated in expression (40).

Then from (56) we obtain the following formula for the average energy density of an ultrarelativistic ideal gas (or a relativistic gas of massless particles)

$$
\rho \approx 3 n k T\left[1+\frac{1}{6}\left(\frac{m c^{2}}{\kappa T}\right)^{2}\right] .
$$

In this limit, from (60) follows the so-called limiting equation of state

$$
P \approx \frac{\rho}{3}
$$

which corresponds to the highest possible pressure at a given average energy density $\rho$.

Based on the above analyzes of the equation of state (60) relating pressure, average energy density and temperature of a relativistic ideal gas, we can write the following approximate equations of state:

$$
P \approx 0, \quad P \approx \frac{\rho}{3},
$$

usually used in the equations of motion of matter (matter and radiation) in cosmological theories [23]. Here, the first equation of state corresponds to a nonrelativistic gas with an ultralow temperature $(T \rightarrow 0)$, the second - to an ultrarelativistic gas with a very high temperature $(T \rightarrow 0)$ or equilibrium radiation. These approximate equations of state used are virtually independent of temperature. Therefore, based on the analysis of the equation of motion of matter, where the equations of state are used, which does not take into account the dependence of pressure and average energy density on temperature, it is impossible, in principle, to determine the change in the temperature of the Universe during its evolution. An additional argument in favor of this conclusion is the fact that the very establishment of the equilibrium Boltzmann distribution and the resulting relativistic distribution of the velocities of gas particles is possible only in statistical equilibrium, with a constant temperature in time. In conclusion, we emphasize that the pressure and average energy density of a relativistic ideal gas take only non-negative values according to their physical meaning. This conclusion remains valid for quantum relativistic ideal gases, since the expressions for the pressure (50) and average energy density (48) of a relativistic ideal gas, as well as the equation of state (52) connecting them, remain valid in the case of quantum statistics. Only averaging follows, it is carried out over the distribution function of the momenta of quantum relativistic ideal gases [24-26]. Thus, no substance can have negative pressure and negative average energy density. This excludes the possibility of the existence of the supposed "dark" matter with such hypothetical properties.

\section{Conclusion}

Let us summarize the consequences of the proposed theory of a relativistic ideal gas, compare them with previously known results, and also discuss issues of their further development. The presentation will be carried out in accordance with the sequence of the issues considered in the article.

1. The distribution function of the particle velocity of a relativistic ideal gas, obtained by us by transforming the distribution function, which is described by expression (13), agrees with the result of Juttner's work [12], obtained by him on the basis of a relativistic generalization of the Gibbs statistic. The "modified" Juttner distribution of velocities obtained in [7] is incorrect, since, according to the authors themselves, it contains a factor - the reciprocal of the energy of a relativistic particle. This leads to the 
erroneous conclusion that in the ultra - relativistic limit the "Modified" Juttner distribution of velocities tends to zero. This contradicts the result (27) and does not correspond to reality (see also a number of curious results given in [7]).

2. The formulas describing the mean and root-mean-square velocity of particles of a relativistic ideal gas were not obtained by either Juttner or his "modifiers". Consequently, for the first time in our work, it was proved on their basis that the characteristic velocities of particles of a relativistic ideal gas are limited - the limiting velocity in nature. This confirms the validity of the fundamental postulate of the special theory of relativity in relativistic statistical physics.

3. The equation of state of a relativistic ideal gas, relating the pressure, average energy density and temperature of a relativistic ideal gas described by expressions (56), (59) and (60), agrees with the results obtained by Juttner [12], as well as other researchers [4, 5, 8-11,16,17]. But unlike these works, in which thermodynamic methods were used to obtain the equation of state (based on free energy, the principle of maximum entropy, etc.), here it was obtained by a new method, namely, by averaging macroscopic quantities for a system of relativistic non-interacting particles, i.e., e. for a relativistic ideal gas from the distribution function of the velocity of their particles. As far as we know, no one has previously used such a method for obtaining the equation of state for a relativistic ideal gas. It is of great interest to generalize the theory of a relativistic ideal gas developed above for reference frames moving with constant acceleration [16], as well as for relativistic plasma of colliding beams [3].

4. Analysis of limit case

A. Substantially Nonrelativistic Ideal Gas $b=\frac{m c^{2}}{k T} \gg 1$.

In this limit, it should be taken into account that

Then we get

$$
E \approx m c^{2}+\frac{P^{2}}{2 m}, k_{2}(b)=\sqrt{\frac{\pi}{2 b}} e^{-b}\left(1+\frac{15}{8 b}\right) .
$$

B. Ultra-relativistic limit.

$$
f \approx \frac{1}{4 \pi} \sqrt{\frac{2}{\pi}} \cdot \frac{1}{(m k T)^{\frac{3}{2}}} \exp \left(-\frac{P^{2}}{2 m k T}\right) .
$$

$b=\frac{m c^{2}}{k T} \ll 1($ or $\mathrm{b} \rightarrow 0)$. Then, taking into account the asymptotics of the function in this limit $\mathrm{k}_{2}(\mathrm{~b})$, we obtain $k_{2}(b) \approx \frac{2}{b^{2}}$. In addition it should be taken into account that

Therefore, we have

$$
E \approx p c \text {. }
$$

Hence it follows that if $\rightarrow 0, f(p) \rightarrow \delta(p)$.

$$
f(p) \approx \frac{1}{8 \pi} \cdot\left(\frac{c}{k T}\right)^{3} \cdot \exp \left(-\frac{p c}{k T}\right)
$$

C. Of the effective cross section of colliding particles

$$
\frac{\sigma}{\sigma_{\max }}=<\sqrt{\left(\overrightarrow{u_{1}}-\overrightarrow{u_{2}}\right)^{2}-\left[\overrightarrow{u_{1}}, \overrightarrow{u_{2}}\right]^{2}}>
$$

Here $u_{1,2}=\frac{u_{1,2}}{c}$. u1,2 - normalized velocities colliding particles of RIG.

Further, the case is considered when, for example, $\mathrm{u}_{2}=0$. Then, the previous formula is greatly simplified.

$$
\frac{\sigma}{\sigma_{\max }}=<u>
$$

Consequently, for parameter values significantly less than one, i.e. for the UR particles, for example, at $\mathrm{b}=10^{-4}$ (which was possible in experiments on particle collisions at CERN, as well as in experiments in Controlled Nuclear Fussion in France), we obtain according to the formula for the average velocity Maxwellian distribution $\langle u\rangle \approx 2 \sqrt{\frac{2}{\pi}} \cdot 10^{2}$.

This result obviously has no physical meaning, since it means that the speed of particles exceeds the speed of light 100 times. But at the same time, according to the formula for the average RIG speed, in this limit we get

$$
\langle u\rangle=1-b^{2}=1-10^{-4}
$$


Consequently, according to the latest results, the effective cross-section of colliding particles of a relativistic ideal gas cannot exceed the effective cross-section of a stationary particle. I.e., starting from a certain threshold energy, the effective cross-section practically does not depend on the average kinetic energy of colliding particles, which is confirmed by experiments conducted at CERN. As an application of the theory of a relativistic ideal gas, one can point out its possible application for calculating the cross section for collisions of ultra - relativistic heavy ions in a high energy collider [27], as well as for the relativistic kinetic description of the production of fermions and bosons in cosmology.

Completing the work, we cannot but pay attention to, perhaps - not very pleasant and annoying, strange circumstance that has developed in the history of the theory of a relativistic ideal gas: for more than a century after Juttner's work [12], they were engaged in an essentially meaningless business - they tried "Modify" ... the correct Juttner velocity distribution! Here, as they say, there is nothing to be done - this is the nature of things. But the truth, although it is bitter, is always fair ...

\section{REFERENCES}

1 Cubero D., et al. Thermal equilibrium and statistical thermometers in special relativity. Phys. Rev. Lett. 2007, Vol. 99, 170601-4.

2 Fanchi J.R. Comparative analysis of Juttner's calculation of the energy of a relativistic ideal gas and implications for accelerator physics and cosmology. Entropy. 2017, Vol. 19, pp. $374-399$.

3 Chason-Acosta G., et al. Manifestly covariant Juttner's distribution and equipartiton theorem. Phys. Rev., E, 2010, Vol. 81, pp. $1-9$.

4 Debbasch F. Equilibrium distribution function of a relativistic dilute perfect gas. Phys. A. 2008, Vol. 387, pp.2443-2454.

5 Schieve W.C. Covariant relativistic statistical mechanics of many particles. Found. Phys. 2005, Vol. 35, pp.1359-1381.

6 Dunkel J., et al. Relative entropy, Haar measures and relativistic canonical velocity distributions. New Journ. of Phys. 2007, Vol.9, pp. $144-158$.

7 Kowalski K., et al. Lorentz covariant statistical mechanics and thermodynamics of the relativistic ideal gas and preferred frame. 2007, arxiv07.12.2725 v.2 [hep-th] 18 Dec.

8 Gonzalez-Narvaez R.E., et al. Mixing of relativistic ideal gases with relative relativistic velocities. Annals of Phys. 2016, 2017 / 01 Vol. 376. pp1-21.

9 Jumaev M.R. Nonlinear fluctuation mechanism for matter creation and reminds of the universe. Sci. Rep. of Bukhara Univ. 2005, No. 1, pp. 66 - 76.

10 Jumaev M.R. Theory of relativistic ideal gas for quasi and ordinary particles. Proc. of the NATO Adv. Res.

Workshop on nonlinear dynamics and fundamental interactions, Kluwer Acad. Publ., Amsterdam. 2006, pp. 155-165.

11 Jumaev M.R. The quantum relativistic ideal gas and of the relict radiation. Lambert Acad. Publ., Germany, 2016, 214 p. [in Russian]

12 Jumaev M.R., et al. Distribution function velocities of the particles relativistic ideal gas. Sci. reports Bukhara Univ. 2020, No. 1, pp. 14-18. [in Russian]

13 Von F. Juttner. Das Maxwellellsche Gefetz in der relative theorie. Ann. d. Phys. 1911, Vol. 34, pp. 856-882.

14 Jumaev M.R., et al. Fluctuational and parametric phenomena in condensed and nanoscopic systems. Abstract Doctor thesis (DSc)., Tashkent, 2021, $72 \mathrm{p}$.

15 Landau. L.D., Lifshitz E.M. Theory of field. Moscow, Nauka. 1988, 512 p. [in Russian]

16 Eichler J. Theory of relativistic ion-atom collisions. Phys. Rev. 1990, Vol.193, pp. 165 - 277.

17 Kaniadakis G. Towards a relativistic statistical theory. Phys. A. 2006, No. 365, pp. 17 - 23.

18 Landau. L.D., Lifshitz E.M. Statistical physics. Pergamon press, 1970, 497p. [in Russian]

19 Van Kampen N. G. Stochastic processes in physics and chemistry. North-Holland, Amsterdam. 2003, 479p.

20 De Groot S.R., et al. Relativistic kinetic theory. North-Holland, Amsterdam. 1980, 355 p.

21 Chuang Liu. Einstein and relativistic thermodynamics in 1952: a historical and critical study of a strange episode in the history of modern physics. Br. J. Hist. Sci. 1992, No. 25, pp. 185 - 195.

22 Hakim R. Introduction to relativistic statistical mechanics, classical and quantum. World Scient. 2011, 295p.

23 Earias C., et al. What is the temperature of a moving body? Scient. Rep. 2017, No. 7, pp. $17657-17600$.

24 Huang Y.S. Relativistic quantum statistical mechanics in the framework of NRT. 2019. Available at: www.researchgate.net/publication/332037148.

25 Nakamura T.K. Relativistic equilibrium distribution by relative entropy maximization. Europhys. Lett. 2008, No. 88, pp. 40009-15.

26 Gradstein I.S., Ryzhik I.M. Tables of integrals, series and products. Acad. Press. 1965, $326 \mathrm{p}$

27 Nakamura T.K. Three views of a secret in relativistic thermodynamics. Progr. Theor. Phys. 2012, No. 128, pp.463-468. 